Revista Mexicana de Astronomía y Astrofísica, Volumen 3, Mayo 1977

\author{
REVIEW OF THE DYNAMIGAL ASPECTS OF \\ TRIPLE SYSTEMS \\ (Invited Paper) \\ V. SZEBE Hely \\ University of Texas at Austin
}

\begin{abstract}
RESUMEN
Se presenta una clasificación de los posibles movimientos de un sistema triple, enfatizando los fenómenos transitorios que ocurren antes de la situación final asintiótica y aclarando la discrepancia entre las formulaciones astronómica y matemática. Se ha descrito la conjetura de una posible inestabilidad y se ha mostrado que los sistemas con energía total negativa y con momento angular bajo, pueden conducir a inestabilidad y a la formación de binarias. La estrella escapada o eyectada puede tener velocidad alta si el encuentro triple que precede al escape es suficientemente estrecho. Los resultados del cálculo de algunas series sistemáticas de tales escapes, se aplican a varias configuraciones estelares.

Se ha revisado el estado actual del problema fundamental de la división del espacio fase cn regiones estables $\mathrm{c}$ inestables y se ha descrito una técnica recién desarrollada aplicable a sistemas estelares. Se señala que las familias de órbitas periódicas recién descubiertas y las configuraciones clásicas previamente establecidas debilitan la conjetura general de inestabilidad.

La posible existencia de sistemas triples en estado de disolución ofrece un intrigante reto observacional para su descubrimiento.
\end{abstract}

\begin{abstract}
A classification of possible motions of triple systems is presented emphasizing the transient phenomena occurring in addition to the final (asymptotic) outcome and clarifying the discrepancies between the astronomical and mathematical formulations. A conjectured possible instability is described and it is shown that systems with negative total energy and low angular momentum may lead to instability and to the formation of binaries. The ejected or escaping star may have high velocity if the triple close approach preceding the escape is sufficiently close. The computational results of several systematic series of such escapes are applied to various stellar configurations.

The present status of the fundamental problem of partitioning the phase-space into stable and unstable regions is reviewed and a recently developed technique, applicable to stellar dynamics is described. Recently discovered families of periodic orbits and previously established classical configurations are shown to weaken the general instability conjecture.

The possible existence of triple systems in states of dissolution offer intriguing observational challenges regarding the discovery of these projected temporary trapezium type systems.
\end{abstract}

\section{REVIEW OF CLASSIFICATIONS OF MOTIONS}

The final (asymptotic) outcome of the motion of triple systems with arbitrary initial conditions has been thoroughly discussed by Chazy (1918-1929) without astronomical implications. His classification has been extended recently to include transient phenomena by Szebehely (1971) and Agekian (1973).
If the system has zero or positive total energy (E) then very soon a Laplacian instability takes over and at least one of the three bodies leaves the system (escapes). The refinements of the classification of this case $(\mathrm{E} \geqq 0$ ) will not be dwelt upon here but rather systems with negative total energy will be emphasized. Note that the amount of angular momentum of systems with $\mathrm{E} \geqq 0$ does not affect the final outcome of the motion qualitatively. 
When the total energy of the system is negative we may have bounded motion, as expected when a (misleading) two-body analogy is recalled, but quite contrary to our two-body experience, unbounded motion may occur also.

The possible bounded motions are:

(1) interplay,

(2) triple close approach,

(3) ejection,

(4) revolution,

(5) equilibrium,

(6) periodic orbits,

(7) oscillation.

The unbounded motions are always associated with binary formations and with the escape of one of the components. (The constrast between negative and positive total energy is that when $E \geqq 0$ at least one body escapes but all three can, while for $\mathrm{E}<0$ such total dissolution of the system cannot occur).

(1) Interplays are known in stellar dynamics as trapezium-type configurations. The system performs a typically three-body motion without any two-body characteristics; for instance, there are no small perturbations and no periodicity, and the motion takes place in a random, possibly ergodic manner. The definition of trapezium-type configurations (Poveda and Allen 1973) does not allow close approaches between two of the members of the triple system since in this case it would belong to the class of hierarchical or classical systems. The distinction between the definitions of "interplay" and "trapezium-type" is the long range behavior reflected by the first and the instantaneous configuration observed by the second. Indeed, if two members of the system have a close approach during interplay then the instantaneous observation will reveal a classical system, but the dynamical character of the motion will be quite different and the future behavior will be chaotic and not classical.

It is well established analytically (Sundman 1912) and numerically (Szebehely and Peters 1967) that binary collisions do not affect the behavior of the system mathematically, i.e., it may be "continued." The Laplacian stability and the triple system will be both terminated from the physical point of view when a collision occurs.
In interplay, binary-type close approaches are allowed but triple close approaches are forbidden for reasons to be discussed below. The essential aspects are boundedness and randomness.

(2) The second type of motion is called triple close approach, which may again be classified instantaneously as trapezium-type configuration. Once again the subsequent motion is crucial since triple close approaches are always followed by ejections and escapes, which, again will be classified (instantaneously) as classical configurations.

(3) By ejection we mean the formation of two two-body systems, consisting of a binary and of an ejected star, moving on an approximately elliptic orbit, relative to the binary. If the ejection orbit is short, this motion might qualify as a trapeziumtype configuration - instantaneously. The ejection becomes an escape if this relative motion is hyperbolic, instead of elliptic. For all practical purposes a long ejection is equivalent to an escape, since the ejected star will probably be lost to its original companions. The angular momentum of the system

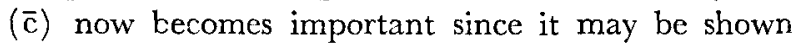
that triple close approaches occur with high probability when $|\bar{c}|$ is small. In turn, ejections and escapes require triple close approaches and therefore systems with low angular momenta have unstable tendencies.

(4) The next type of motions are called revolutions in dynamics and are known as classical systems in stellar dynamics. From Harrington's (1967-1975) thorough research we know that as long as the semi-major axis of the inner binary $\left(a_{1}\right)$ is smaller than the periastron distance of the outer orbit $\left(q_{2}\right)$ by a factor of approximately 3 , this motion shows remarkable stability. Eccentricities, masses, orbital inclinations and phases might influence this factor since it is known that certain combinations result in a triple close approach and in consequent instability and escape.

(5) The class called equilibrium is associated with Lagrange's name and is also known as librational motion. It should be recalled that the triangular equilibrium solutions are stable in the Solar System. Indeed, many Trojan asteroids exist, but when the participating masses are about equal, all five equilibrium solutions become unstable.

(6) The class of periodic orbits received recently the increased attention they deserve. This belated 
acceptance of Poincaré's advice was made possible by increased efficiency of electronic computing machines, since numerical integration of the 18th order system of differential equations describing the general problem of three bodies by hand, seems to be impractical, even if the issue is only the computation of one single trajectory. The performance of differential corrections necessary to find periodic orbits and the essential associated stability calculations, in addition to the numerical integrations, presented serious practical computational difficulties when families of periodic orbits were to be established. Some of these families of periodic orbits possess relative periodicity, i.e., they are periodic only with respect to a rotating frame as shown by Hénon (1974) and Hadjidemetriou (1975a) but this does not restrict either their validity or their importance. The existence of the families of stable periodic orbits suggests non-zero measure for bounded motions since the perturbed stable periodic motions should lead to quasi-periodic orbits which are bounded. Such families of periodic orbits are generated today by using several ideas. An interesting method favored by Hadjidemetriou (1975b), Bozis and Christides (1975), Broucke (1975) and Dunham (1975) is to use the families of periodic orbits of the restricted problem as generators for periodic orbits in the general problem by increasing the mass of the third body (not one of the primaries). In this way, every single member of a family in the restricted problem gives rise to a family (with variable mass) of the general problem. This leads us, therefore, to a picture somewhat overwhelming regarding the denseness of periodic orbits in the phase-space. The study is on its way and the organization of the large number of orbits is expected to be a serious task. Once again, the determination of the stability properties of these orbits seems to be the most important aspect. The essential question is connected with the stability of the above classes of motions as well as with their probability of occurrence.

(7) In this astronomically oriented review we omit the discussion of oscillatory motions because of their purely mathematical interest.

A few remarks regarding the relation between the theoretical and observational results might be in order at this place. The presently existing, admittedly incomplete, numerical and analytical results show that the stable systems (revolution and stable periodic orbits) are not favored by the computer. In other words, using random initial conditions neither of these classes appear with significant frequency as the outcome of numerical integrations. The only class with Laplacian instability (escape) seems to occur with high probability on the computer, especially with small values of the angular momentum.

On one hand, therefore, we have the existence of families of stable periodic orbits indicating that, at least in certain ranges of the phase-space, escapes are improbable. Furthermore, the classical systems appear to be stable in nature as well as on the computer. These systems also occupy parts of the phase-space where escapes are rare. On the other side of the ledger we must list the large regions of the phase-space in which the escape orbits are located. Since the, as yet unobserved, systems in the process of escape are highly unstable they represent observational challenges. During the early stages of the escape process, the instantaneous picture is that of a trapezium-type configuration, while later on the possibly misleading temporary observation would indicate a classical system. In turn, classical or trapezium-type systems might indeed by escaping systems unless proven otherwise, especially since numerical results indicate that escapes occur with high probability. The observed and verified classical systems represent theoretical challenges since the formation of such systems, using three-body point-mass models seems to be improbable. The origin of classical triple systems is connected with either systems of higher multiplicity or with triple systems which at formation possess sufficient angular momentum and special dynamical conditions (selective initial conditions). The origin of escaping systems requires low angular momentum and random initial dynamic specifications.

Regarding the discrepancies between observations, theories, and interpretations, a well known example related to the restricted problem of three bodies might be recalled. Lagrange in 1772 proved the existence of five equilibrium points as solutions of the restricted problem of three bodies formed by the Sun and Jupiter as primaries. Not until 1906 was the first Trojan asteroid (588 Achilles) discovered in the neighborhood of one of the triangular 
equilibrium points. Theoreticians cherish this example demonstrating that analysis might sometime assist observations. It should be pointed out that astronomical photography started only in 1892 (Gingerich 1975 ), therefore, 120 years of the total 134 years of delay between Lagrange's announcement and the discovery of Achilles may be contributed to insufficient technology. It is interesting to contemplate as an imperfect analogy a similar theoretical prediction, according to which, as yet unseen phenomena (escapes from triple systems) should be eventually observed. The analogy with Lagrange's accomplishment is weak and incomplete since his physically realistic models and precise mathematical result are replaced here by conjectures based on qualitative semi-analytical theories and numerical experiments.

\section{PARTITIONING OF THE PHASE-SPACE}

The establishment of the list of possible motions of triple systems is only the first step in the process of predicting the probable outcome of the motion. The task of partitioning the phase space consists of giving qualitative and if possible also quantitative description of the motions associated with all points of the phasespace.

The 18th order system of differential equations may be reduced to a 6 th order system for the threedimensional general problem of three bodies. Such reductions eliminate the time and yield rather complicated equations of motions without further integrals.

The 12th order system of differential equations representing the general planar problem of three bodies might be similarly reduced to the fourth order. If no use is made of the integral of energy and if the time is not eliminated, the process of reduction may be terminated with a 6 th order system as shown by Whittaker (1904). Let

$$
\mathrm{H}=\mathrm{H}\left(\mathrm{q}_{1}, \mathrm{q}_{2}, \mathrm{q}_{3} ; \mathrm{p}_{1}, \mathrm{p}_{2}, \mathrm{p}_{3}\right)
$$

be the Hamiltonian function of this system, with a positive definite Hessian, $\partial^{2} \mathrm{H} / \partial \mathrm{p}_{\mathrm{i}} \partial \mathrm{p}_{\mathrm{j}}$.

The equations of motion are

$$
\dot{\mathrm{q}}_{\mathrm{i}}=\frac{\partial \mathrm{H}}{\partial \mathrm{p}_{\mathrm{i}}} \text { and } \dot{\mathrm{p}}_{\mathrm{i}}=-\frac{\partial \mathrm{H}}{\partial \mathrm{q}_{\mathrm{i}}}, \quad \mathrm{i}=1,2,3 \text {, }
$$

therefore, the equations of zero velocity are given by

$$
\frac{\partial H}{\partial p_{i}}=F\left(q_{i}, p_{i}\right)=0
$$

Solving these equations for $p_{i}$ and substituting in the Hamiltonian, we have

$$
\overline{\mathrm{H}}\left(\mathrm{q}_{1}, \mathrm{q}_{2}, \mathrm{q}_{3}\right)=\mathrm{E}
$$

Note that the constant $\mathrm{E}$ is the total energy and the function $\mathrm{H}$ contains the angular momentum as a parameter. Consequently, we have as the equation of the surfaces of zero velocity:

$$
f\left(q_{1}, q_{2}, q_{3},|\bar{c}|, E\right)=0 .
$$

These surfaces of zero velocity may be constructed (Szebehely and Zare 1975, Zare 1976) using the above equation or may also be established by means of Sundman's inequality (Marchal 1975, Marchal and Saari 1976). These manifolds are presently analyzed in detail but a few essential facts are becoming already clear.

The surfaces are not closed, consequently bounded motion might occur but it does not have to occur. If the surfaces would be bounded for some values of $|\bar{c}|$ and $E$ it would mean that in those regions escape is impossible.

Another result of interest is that the pertinent parameters, as mentioned before, are the total energy and the angular momentum of the system. In fact, the product, $|\mathrm{E}| \overline{\mathrm{c}}^{2}$ is invariant regarding changes in scale and therefore, it is a useful (but not a dimensionless) parameter.

The combined effect of $|\mathrm{E}|$ and $|\overline{\mathrm{c}}|$ is rather complicated. It is known that when $\mathrm{E}=0$, escape occurs independently of the value of $|\overrightarrow{\mathrm{c}}|$. It is also known that the occurrence of close triple approaches are enhanced by small values of $|\overrightarrow{\mathrm{c}}|$. When a symmetric total collapse (with $|\overline{\mathbf{c}}|=0$ ) is perturbed with small asymmetries introduced by small velocities the slight increase of $|\overline{\mathrm{c}}|$ results in very fast escapes. As $|\bar{c}|$ increased further, these escapes become ejections and eventually interplays. Meanwhile, the total energy of the system $|\mathrm{E}|$ is decreasing since the initial configuration is kept fixed and the initial velocities are increased. This process of 
$|\mathrm{E}| \rightarrow 0$ does not affect much the outcome of the motion and the value of $|\overline{\mathrm{c}}|$ controls the behavior of the system until $|\mathrm{E}| \simeq 0$. Such a process is described by Szebehely (1974).

The system of three bodies from the point of view of dynamics is reversible since the motion returns to the initial conditions if at any time the velocities (or the time) are reversed. From the point of view of stellar dynamics an escape (or for that matter a long ejection) is an irreversible process since the escaping star will not return to its original initial conditions. We refer to such irreversibility as physical. So when the symmetry and the periodicity of the above described triple collision is broken, physical irreversibility follows. This idea of the asymmetry as a physical reversibility breaking process is also operational for the symmetric equilibrium solutions as well as for their continuations to the well known librational motions. When the symmetry and the periodicity of these solutions is broken, physical irreversibility sets in. Some numerical results, scaled to stellar systems might be of interest at this point. These examples are based on the idea that escapes (or binary generations) are usually preceded by triple close approaches. These triple close approaches must possess sufficient asymmetry so that the distances occurring will be realistic and that the assumption of Newtonian gravitational forces acting between point masses will not be violated. That is, since for instance tidal effects are not included in these calculations the lower limit of allowable distances should be about 100 or 1000 times the radii of the participating bodies. For simplicity the three masses are assumed to be equal in these examples though interesting modifications of the results appear when this assumption is dropped. The method of generation of the triple close approach is not important, since it does not influence the escape. As long as two of the stars have a close approach (perturbed periastron) while the third star is not too far and it is moving toward the two of them, such triple close approach will result in an escape with high probability. (It should be repeated at this point that random initial conditions with low angular momentum and negative total energy, in most of the cases will lead eventually to a triple close approach.)
Consider then a close approach $\left(R_{\min }\right)$ between two stars, preceding the triple close approach: At the time of periastron the relative velocity is $V_{\max }$. After the triple close approach the binary formed possesses a semi-major axis (a) and the third body escapes with an escape velocity of $\mathrm{V}$.

In the first example the motion of three solar type stars is investigated. Let the first close approach be $R_{\min }=0.1 \mathrm{AU}$ (or $20 \mathrm{R}_{\odot}$ ) with $\mathrm{V}_{\max }=184 \mathrm{~km} \mathrm{~s}^{-1}$. The escape velocity is $\mathrm{V}=23 \mathrm{~km} \mathrm{~s}^{-1}$ and the semimajor axis of the binary left behind is a $=1.2 \mathrm{~A} \mathrm{U}$. If the closest approach is $R_{\min }=10 \mathrm{AU}$, the semimajor axis becomes $\mathrm{a}=120 \mathrm{AU}$, the maximum velocity is $V_{\max }=18.4 \mathrm{~km} \mathrm{~s}^{-1}$ and the escape velocity decreases to $\mathrm{V}=2.3 \mathrm{~km} \mathrm{~s}^{-1}$.

If the participating bodies have higher densities the minimum distances allowed might be decreased. Combination of various types of stars might be considered at this point. High density white dwarfs, if themselves or if in combination with other types of stars create a close triple configuration, the escape velocity may be as high as $\mathrm{V}=280 \mathrm{~km} \mathrm{~s}^{-1}$ or as low as $\mathrm{V}=28 \mathrm{~km} \mathrm{~s}^{-1}$ depending on the closest distance $\left(R_{\min }=10^{5} \mathrm{~km}\right.$ and $\left.R_{\min }=10^{7} \mathrm{~km}\right)$ allowed.

Not necessarily as an astronomical reality, but for completeness sake it might be mentioned that if neutron stars would participate, escape velocities as high as $6100 \mathrm{~km} \mathrm{~s}^{-1}$ might be computed.

Regarding galaxies with $10^{10}$ solar masses, the escape velocities become $V=343 \mathrm{~km} \mathrm{~s}^{-1}$ if $R_{m \text { ln }}=$ $1000 \mathrm{kpc}$ or $\mathrm{V}=108$ if $R_{\mathrm{min}}=10^{4} \mathrm{kpc}$. The corresponding semi-major axes are $\mathrm{a}=1.2 \times 10^{4} \mathrm{kpc}$ and $1.2 \times 10^{5} \mathrm{kpc}$.

The above computations are based on papers by Szebehely (1974). It may be added that the computational results may be verified analytically and it may be shown that as the triple close approach becomes closer and closer, the binary formed becomes more tight and the escape velocity becomes higher. In fact in the previously discussed cases $V^{2} \mathrm{a}=$ constant and also $\mathrm{V}^{2} \mathrm{R}_{\mathrm{min}}=$ constant. Therefore, if arbitrary close approaches are allowed $\left(R_{\min } \rightarrow 0\right)$ the escape velocity theoretically increases without limit $(\mathrm{V} \rightarrow \infty)$; see also Waldivogel (1975).

\section{SUMMARY}

(1) Stable triple systems of the classical type are probably formed from systems of higher multiplicity 
of trapezium-type or from triple systems of high angular momenta.

(2) The low angular momentum triple systems lead to escapes, offering an outstanding possible mechanism for binary formation.

(3) The quantitative and qualitative research on periodic orbits and on classical systems indicates that at least some parts of the phase-space are occupied by bounded motions.

(4) The facts that the surfaces of zero velocity are open and that random initial conditions, especially for low values of the angular momentum lead to escape, indicate that it is not unreasonable to conjecture that escape is the dominant mode of behavior.

\section{REFERENCES}

Agekian, T. A., and Martinova, A. I. 1973, Unin. of Leningrad Pub., No. 1, 122.

Allen, G., and Poveda, A. 1974 Proc. IAU Symposium No. 62, The Stability of the Solar System and of Small
Stellar Systeris ed. Y. Kozai (Dordrecht: D. Reidel), 239.

Birkhoff, G. D. 1927, Dynamical Systems (Providence, R. I.: Am. Math. Soc. Pub.).

Bozis, G., and Christides, Th. 1975, Celes. Mech., 12, 277. Broucke, R. 1975, private communication.

Chazy, J. 1918, Bull Astron., 35, 311.

Chazy, J. 1929, J. Math. Pure Appl., 8, 353.

Dunham, J. 1975, private communication.

Gingerich, O. J. 1975 , private communication.

Hadjidemetriou, J. 1975a, Celes. Mech., 12, 255.

Hadjidemetriou, J. 1975b, Celes. Mech., 12, 155.

Harrington, R. S. 1968, A.J., 73, 190.

Harrington, R. S. 1975, Celes. Mech., in press.

Hénon, M. 1974, Celes. Mech., 10, 375.

Marchal, C. 1976, Long-Time Predictions in Dynamics, eds. V. Szebehely and B. Tapley (Dordrecht: D. Reidel), 181.

Marchal, C., and Saari, D. 1975, Celes. Mech., 12, 115.

Standish, E. M. 1970, in Periodic Orbits and Stability, ed. G.E.O. Giacaglia, (Dordrecht: D. Reidel), 375.

Sundman, K. F. 1912, Acta Math., 36, 105.

Szebehely, V. 1971, Celes. Mech., 4, 116.

Szebehely, V. 1974, A. J., 74, 981-1449.

Szebehely, V., and Peters, F. 1967, A. J., 72, 1187.

Szebehely, V., and Zare, K. 1976, Bull. $A A S, 8,436$.

Whittaker, E. T. 1904, Analytical Dynamics, (London and New York: Cambridge Univ. Press).

Zare, K. 1976, Dissertation, University of Texas at Austin.

\section{DISCUSSION}

Huang: I was greatly impressed by Professor Szebehely's very general and elegant treatment of the three-body problem. I am especially interested in the zero-velocity surface. The openness of the zero-velocity surface, while derived mathematically, may be interpreted physically, if you permit me and please correct me if I am wrong, by the fact that there is an infinite energy reservoir between two stars, or a singularity, as theoreticians prefer to call it. This can supply energy for the third body to escape. In a sense this is like the nuclear fusion; only the energy that could be liberated in this case is infinite. Such a large amount of energy can do a lot of mischievous things to make people who are working in this field uncomfortable.

Szebehely: Thank you. I am in complete agreement.

Evans: I think the statistics of observed spectroscopic binaries allow you to have distinctly smaller values of $R$.

Szebehely: Using $R_{\min }=2 R_{\odot}$, the semi-major axis of the binary becomes $a=0.12 A U$, the escape velocity is $\mathrm{V}=73 \mathrm{~km} / \mathrm{sec}$, and the maximum velocity is $V_{\max }=582 \mathrm{~km} / \mathrm{sec}$. Poveda: Your distance of minimum approach, $R_{\mathrm{min}}=0.1 \mathrm{AU}$ for solar-type stars, seems too large; perhaps $R_{\min }=0.01 \mathrm{AU}$ will be more appropriate. On the other hand, for galaxies $\mathrm{R}_{\min }$ seems too small, due to the large sizes of galaxies.

Szebehely: Using $\mathrm{R}_{\mathrm{min}}=0.01 \mathrm{AU}=2 \mathrm{R}_{\circ}$, we obtain the numbers given in $\mathrm{my}$ answer to Dr. Evans' question. Regarding the examples concerning galaxies, we might wish to use $R_{\mathrm{min}}=1000 \mathrm{kpc}$, corresponding to $\mathrm{V}=343 \mathrm{~km} / \mathrm{sec}$.

Scarfe: The tidal effects producing apsidal rotation in solar-type stars seem to be important around $0.02-0.05 \mathrm{AU}$, depending on the stellar masses.

Szebehely: Thank you. See my reply to Dr. Evans' question. 